\title{
Refinement of the calculation of the parameters of the kinetics of grinding plant raw materials in a vibration mill (based on mathematical models)
}

\author{
N. D. Orlova \\ National University "Odessa Maritime Academy" Odessa, Ukraine \\ Corresponding author E-mail: nator12969@gmail.com
}

Paper received 07.09.20; Accepted for publication 24.09.20.

\begin{abstract}
https://doi.org/10.31174/SEND-NT2020-238VIII29-13
\end{abstract}
\begin{abstract}
The process of destruction of plant raw materials is considered from the point of view of the evolution of the interfaces of individual structural elements with the further development of cracks on these surfaces. The emergence and development of cracks is considered from the point of view of fractal geometry as the formation of a fractional-dimensional layer. The article proposes formulas for calculating the specific energy saturation of the dispersed system and the specific surface of soil material using the representations of fractal geometry.
\end{abstract}

Keywords: grinding of vegetal raw materials, specific energy saturation, specific surface area, fractal dimension.

The task of the theory of vibration grinding is to establish the relationship between the fineness of the product obtained, physicochemical and mechanical properties, energy consumption and the parameters of a vibration mill. For the practical use of vibration mills, it is important to foresee the technological result of vibration impact on plant raw materials of various properties. Plant raw materials belong to the category of materials that are difficult to grind, since in the bulk they have a low mass of solid particles and a strong fibrous structure and represent a complex natural structure.

The main requirement for devices for processing plant materials is the preservation of the chemical and physical properties of the processed material. Depending on the nature of plant material and the nature of its use, specific requirements are imposed on grinding, in terms of dispersion, purity, preservation, etc. There are two types of grinding, depending on the size of the starting material and the final product

- crushing (large - from 1000-200mm to 200-50mm; medium - from $250-50 \mathrm{~mm}$ to $40-10 \mathrm{~mm}$; small - from 50 $25 \mathrm{~mm}$ to $10-1 \mathrm{~mm})$;

- grinding (fine - from $25-3 \mathrm{~mm}$ to $1-0.4 \mathrm{~mm}$; colloidal from $0.2-0.1 \mathrm{~mm}$ to $0.001 \mathrm{~mm}$ ).

Vibratory mills are used for ultrafine grinding with a particle size of approximately $10 \div 0.1 \mathrm{~mm}$ to 0.00075 $0.0001 \mathrm{~mm}$.

In most cases, the task is to obtain the finest powders as possible, subject to the limitation of time and energy consumption, which leads to the need to study the laws of the process of fine and ultrafine grinding.

The main theoretical result of works $[1,2,4,5]$ on the study of the process of grinding plant materials is the consideration of various mathematical models (energy and probabilistic), which at certain stages quite accurately describe the processes under consideration.

In the energy model, the efficiency of the process according to [3] is estimated by the specific energy saturation of the dispersed system, accumulated in the volume of the ground material, and is expressed by the dependence

$$
\mathrm{E}_{\mathrm{yd}}=\frac{1}{2} \eta \frac{1}{\mathrm{~V}_{\mathrm{K}}} \frac{\mathrm{dW}}{\mathrm{dt}} \frac{\mathrm{P}}{\mathrm{v}_{\mathrm{m}}}
$$

where is $\eta$ - the coefficient of energy absorption by the dispersed system during grinding
( $\eta<1) ; \quad V_{K}-$ the volume of the crushed material; $\mathrm{U}_{\mathrm{m}}-$ speed of movement of grinding bodies; $\mathrm{W}-$ kinetic energy of grinding bodies; $\mathrm{P}$ - characterizes the strength properties of the crushed material.

In the probabilistic model [2, 5], the dispersion characteristics of plant materials are used, which characterize both an individual particle and the entire ensemble of particles. A particle and its size (linear) and shape (volumetric characteristics) are characterized by the specific surface of a particle or an ensemble of particles $S_{\div}$. For a non-porous particle of any shape, the definition of volume is quite unambiguously determined $V=\frac{P}{\rho}$ (the ratio of weight to density of the material).

The volume of the unit is taken as the volume of the space bounded by the outer surface.

The choice of a mathematical model of the powder to be ground depends on the goals of the researcher, but in each of the models, the calculation of parameters ( $\mathrm{E}_{\text {yd }}, S_{\div}$etc.) depends on the volumetric (linear) characteristics of particles or an ensemble of particles.

For a porous particle of plant origin, the concept of volume is somewhat modified. A porous particle as an aggregate of non-porous particles is modeled with a Menger sponge [6,7,8]. Menger's sponge (fig.1) is formed as follows. We will assume that an aggregated porous particle is a cube with a side length equal to $X$. The side of the original cube is divided into $k$ parts, after which cubes with an edge $\frac{X}{k}$ side are removed from the center of the cube $\boldsymbol{m}$. The proportion of the remaining ones is calculated and the withdrawal procedure continues once $n(n \rightarrow \infty)$, the number $N$ ) of cubes of the scale $\frac{X}{k^{n}}$ is equal

$$
N=\left(k^{3}-3 k m^{2}+2 m^{3}\right)^{n}
$$




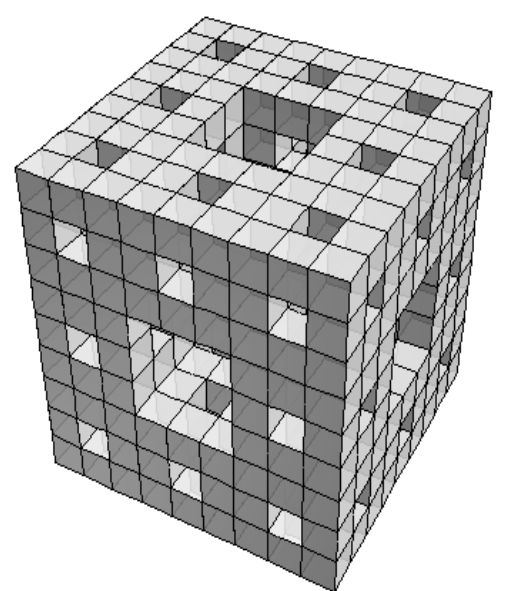

Fig.1

Menger sponge volume

$V_{j}=X^{3} \cdot 3^{n \cdot D_{1}}$

where $\quad D_{1}=\frac{\ln \left(k^{3}-3 k m^{2}+2 m^{3}\right)}{\ln k}$ is the fractal

dimension of the generalized Menger sponge; $D_{1}$ - fractal dimension of the space occupied by a porous particle (Menger sponge)

$$
2 \prec D_{1} \prec 3 ; D_{1}=\frac{\ln 20}{\ln 3}=2,7268 \text {. }
$$

In this case, porosity is understood as the total fraction of the withdrawn cubes from the original cube of volume $X^{3}$. Note that the term "fractal" was introduced into scientific consideration by the fundamental works of mathematician Benoit Mandelbrot in the mid-70s of the last century when describing the shape of complex natural structures.

It is known that $[1,4,5]$ finely dispersed material aggregates, and the particles of the crushed material are collected in aggregates that behave as a whole (particle). In a chemical or sorption process, the inner surface of the aggregates takes part in this process, and a particle in this case is only a part of the solid material, limited by the liquid or gaseous phase.

An aggregated particle can consist of particles of dissimilar materials, which disintegrate only with sufficient mechanical or other type of action. Using the concept of fractal [8], you can build more realistic models of porous systems.

The direct and most used methods of analysis of variance include sieve analysis. Granulometric composition is the most complete dispersed characteristic of a powder; however, for practical purposes, the specific surface area $S_{\div}$is more convenient.

The specific surface area of a particle is the ratio of its total surface area or external surface area (for aggregates) to its weight. Characterization of non-porous particles with linear dimensions (diameter) is possible only if they are geometrically correct particles (ball, cube). For porous particles, the linear size can also be introduced using fractal geometry (analogue of the Koch curve) $[6,8]$

$X_{j}=X \cdot 3^{n(D-1)} D=\frac{\ln 4}{\ln 3} \approx 1,26$

The dispersion of the powder $[1,2,5]$ is the characteristic of the size (shape) of the particles making up the powder. If the particle is porous, then the concept of surface area for it can also be introduced using fractals

$S_{j}=\frac{X j^{2}}{m}, \quad S_{j}=\frac{X^{2} \cdot 3^{2 n(D-1)}}{m}$

$m=1$

where $n$-is the number of pores; the classic Menger sponge corresponds $k=3, m=1$ to i.e. the case of a small fraction of pores in this case $D \approx 1,26$.

With the help of the considered concepts, let us clarify the most common formula for determining the specific surface $[1,2,5]$

$\mathrm{S}(\mathrm{t})=\frac{\mathrm{k}(\mathrm{t})}{\gamma(\mathrm{t}) \overline{\mathrm{X}}(\mathrm{t})}$

where $k-$ is the coefficient equal to the ratio of the surface area to the volume of the particle; $\gamma(\mathrm{t})-$ bulk weight; $\bar{X}(t)-$ is the average particle size per unit weight.

For ideal powder particles having the shape of a cube (ball) according to $[1,2] k=\frac{6}{X}$

where $X$ is the length of the edge of a cubic particle.

However, powder particles rarely approach the shape of a cube (ball), so the value of the coefficient is determined experimentally and depends on the size of the particles and the material being ground. So according to [1.5] for particles with size $-\bar{X}(\mathrm{t}) \succ \mathbf{1 0 0 0 i ̀ ~ e ̂ i ́ ~ - ~}$ $\mathrm{k}=\mathbf{4} \div \mathbf{8} ; \overline{\mathrm{X}}(\mathrm{t}) \leq \mathbf{1 0 0 i ̀ ~ e ̂ i ́ ~}-\mathrm{k}=\mathbf{1 . 5} \div \mathbf{2}$.

In this calculation of the coefficient, the presence of internal surfaces in cracks and fractures of particles is not taken into account, which significantly affects the value of the specific surface. Taking into account formula (2), to calculate the coefficient $k$, we can propose the formula

$\mathrm{k}=\frac{6}{\mathrm{X} \cdot 3^{\mathrm{n}(\mathrm{D}-1)}} \quad \mathrm{D}=\frac{\ln 4}{\ln 3} \approx \mathbf{1}, 26$

The value of the coefficient $\mathrm{K}$ can be further specified using formulas (1), (3).

In this case, the value of the coefficient $k=\frac{S_{j}}{V_{j}}$ equal to the ratio of the surface area to the volume of the particle is proposed to be calculated by the formula

$\mathrm{k}=\frac{3^{2 \mathrm{n}(\mathrm{D}-1)}}{\mathrm{X} \cdot 3^{\mathrm{D}_{1} \cdot \mathrm{n}}}$

where $\mathbf{n}-$ is the number of pores.

The formula for calculating the specific surface area of plant materials takes the

$\mathrm{S}(\mathrm{t})=\frac{3^{2 \mathrm{n}(\mathrm{D}-1)-\mathrm{n} \cdot \mathrm{D}_{1}}}{\gamma(\mathrm{t}) \cdot \mathrm{X} \cdot \overline{\mathrm{X}} \cdot \mathrm{t})}$

In the energy model, the process efficiency formula takes the form

$E_{y d}=\frac{1}{2} \eta \frac{1}{X^{3} \cdot 3^{n \cdot D_{1}}} \frac{d W}{d t} \frac{P}{v_{m}}$

Thus, the use of the concept of fractal allows one to 
build more realistic models of porous systems; more accurately determine the parameters of processed plant materials in various mathematical models.

\section{REFERENCES}

1. Nepomnyashchy E.A. Regularities of fine grinding, accompanied by aggregation of particles. / E.A. Nepomniachtchi // Theoretical Foundations of Chemical Technology. - 1978.v.12. No. 4 - S. 576-580.

2. Ovchinnikov P.F. Vibrorheology / P.F. Ovchinnikov // - Kiev: Naukova Dumka - 1983. - 271p.

3. Oshkordin O.V. Kinetics and dynamics of grinding plant raw materials for food production / O.V. Oshkordin, L.Yu. Lavrov, G.A. Usov // Polzunovsky Bulletin. -Published: Altai State Technical University I.I. Polzunova (Barnaul) -2011. -№2 / 2. -S. 202-206. ISSN: 2072-8921.

4. Kulikov VD The physical nature of destruction / VD Kulikov, NV Merkalova, M.M. Zakrinichnaya // Under the general editorship of Professor Kuzeev I.R. - Ufa - 1999.- 207C.

5. Kafarov VV System analysis of processes of chemical technology: methods of unequal thermodynamics: monograph / IN Dorokhov, EM Koltsova; otv. editor N.M. Zhavoronkov - 2nd ed. rev. and add. - Moscow: Yurayt Publishing House, 2018.- 377 pp .- (Series: Universities of Russia) ISBN 9785-534-06997-6.

6. Orlova N.D. Geometric and physical interpretation of some mathematical concepts / Orlova N.D. // Conference materials Theory and practice of grinding and separation processes Odessa 2001. - P.159-166.

7. Fist M.I. Structural aspects of fractal mechanics of woodpolymer composites / M.I. Fist // Izv. Academy of Sciences of the BSSR. Ser. physical and technical nauk - 1991. - N 2 P.18-22.

8. Mandelbrot B. Self-affine fractal sets // In the book "Fractals in Physics" - M .: Mir - 1988. - 672P. 